\title{
Assessing The Conservation Value of Farmland Ponds: Use of Predictive Techniques to Identify Surrogate Groups
}

\author{
GIORIA M*, BACARO G, SCHAFFERS A
}

Margherita.gioria@ucd.ie

School of Agriculture, Food Science, and Veterinary Medicine University College Dublin 


\section{Freshwater biodiversity - Ponds}

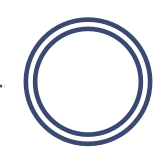

- Ponds (i.e. water bodies comprised between $25 \mathrm{~m}^{2}$ to $2 \mathrm{ha}$ ) are among the most diverse and yet threatened freshwater ecosystems

- More species, more uncommon, rare, and threatened species compared to lakes, rivers and streams (ditches)

- Biodiversity hotspots

- Pond conservation = cost-effective opportunity to maintain and enhance biodiversity

○ Small size catchment area

○ Large number

- Model systems in biology and ecology 


\section{Effective pond conservation}

Effective pond conservation requires:

1. The conduction of large-scale pond biodiversity inventories

2. The development of long-term monitoring programmes aimed at assessing changes in pond distribution and biodiversity associated with socio-economic and environmental changes

3. Identifying the drivers of pond biodiversity

4. A classification system for ponds based on the biotic communities they support and on environmental and management variables 


\section{Surrogate groups}

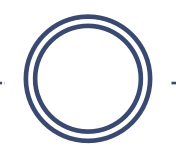

- Limited resources to undertake biodiversity surveys

- Increasing interest in identifying surrogate taxa to be used as indicators of biodiversity

- Cross-taxon congruence $=$ relationship between (among) taxonomic groups

- More effective than environmental surrogates 


\section{Cross-taxon congruence analysis - 1}

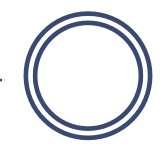

- Def = relationship between (among) taxonomic groups

- Consistent correlation (spatial scale)

- Similar response to environmental variables

- 3 main mechanisms to explain congruence between pairs of taxa (Gaston \& Williams, 1996) :

$\circ$ 1) trophic, competitive, or mutualistic interactions between taxa;

○ 2) common determinants of community patterns;

$\circ$ 3) random draw of taxa from a larger pool of available colonists 


\section{Cross-taxon congruence analysis -2}

- If there was a strong and consistent correlation between two or more taxonomic groups

$\rightarrow$

- We could use of one taxonomic groups to predict community patterns for other groups

- This would allow:

○ Rapid pond biodiversity assessments

o Identify ponds of high conservation value (hotspots)

$\circ$ Monitoring programs 


\section{Pond biodiversity indicators}

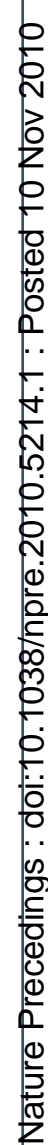

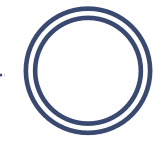

$\square$ Wetland plants

口 Taxonomy and ecology well-known

口 Sensitive to environmental changes

$\square$ Water beetles

口 Speciose (244 species in Ireland)

口 Predaceous diving beetles: multispecies assemblages

口 Ubiquitous 


\section{Objectives}

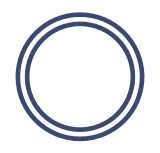

- Quantify the relationship between the vegetation and water beetles

- Evaluate the power of vegetation data in predicting patterns in patterns in water beetle assemblages

- Biotic-abiotic relationships for both plant and beetle assemblages 


\section{Biotic and environmental data}

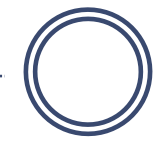

- 54 ponds (June/July 2008 and 2009)

○ Water beetle data (counts)

○ Wetland plant data (\% cover)

- Environmental variables

o Size (surface area, max depth, max depth of sampling)

$\circ$ Permanency $(\mathrm{T} / \mathrm{P})$ *

$\circ$ Grazing intensity $(\mathrm{U}, \mathrm{G}, \mathrm{F})$ *

○ Substratum $(\mathrm{Gv}, \mathrm{M})$ *

$\circ$ Pond age $(2-10 ;>10$ years $)$ *

○ Physico-chemical variables

o $\mathrm{NO}_{3}-\mathrm{N}, \mathrm{PO}_{4}-\mathrm{P}, \mathrm{NH}_{3}-\mathrm{N}, \mathrm{pH}$, alkalinity, $\mathrm{DO}$, conductivity 


\section{Data analysis}

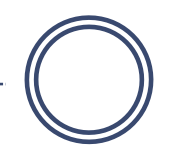

- Cross-taxon congruence (community concordance or correspondence)

○ Species richness

- Species composition

- Biotic-abiotic relationships

○ Species richness

○ Species composition 


\section{Cross-taxon congruence}

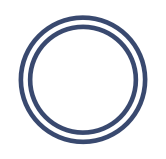

- Species richness

- simple regression analysis (Pearson correlation)

- Species composition (counts - \% cover):

○ Mantel tests

o Procrustes analysis - PROTEST (Gower 1971, Jackson 1995): 1) original data; 2) NMDS

○ Co-correspondence analysis (Co-CA; ter Braak \& Schaffers 2004) r Predictive - symmetric

o Similarity percentages analysis (SIMPER, Clarke 1993) 


\section{Mantel tests}

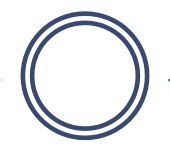

Commonly used in ecology to evaluate the correlation between two data matrices

- 1) Standard parametric approach (Pearson correlation)

$\circ$ assesses the degree of correspondence between two distance/dissimilarity matrices calculated for the plant and beetle datasets;

0 the significance of the relationship between the plant and beetle distance matrices was tested using 10,000 Monte Carlo randomizations (Jackson 1995; Manly 1997)

- 2) A non-parametric Mantel approach based on the Spearman rank correlation (Clarke 1993; 10,000 permutations) 


\section{Procrustes analysis - 1}

- This is a multivariate ordination technique (Gower 1971)

- It uses a rotational-fit algorithm to maximize the sum-of-square distances between corresponding points of two data matrices

- Procrustes analysis fits one matrix to another by rotating, translating, and rescaling one matrix to minimise the sum-of-squared residuals between two matrices

- The goodness of fit measure between these matrices is the $m^{2}$ sum-ofsquared deviation statistic (Gower 1971)

- $m^{2}$ is a measure of concordance when two matrices

- The Procrustes approach can be performed using either raw data matrices or the results of direct or indirect gradient analyses 


\section{Procrustes analysis -2}

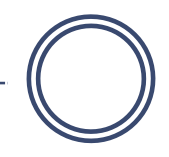

- The significance of the Procrustean fit $\left(m^{2}\right) \rightarrow$ randomization or bootstrapping tests (Jackson 1993; Husson et al. 2009)

- Advantage:

- Based on the original matrices or ordination

- Higher power than Mantel tests (Pere-Neto \& Jackson 2001)

○ Consistent - Pearson correlation

- The use of the results of multivariate analyses (CA, NMDS - PCA) of the original dataset is useful to reduce the data dimensionality

- PROTEST (software)- R ('shapes', 'FactoMineR', ....) 


\section{Co-correspondence analysis}

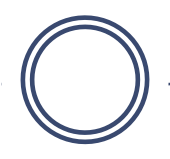

- ter Braak \& Schaffers (2004); Schaffers et al. (2008)

- Direct approach - 2 biological matrices

- Predictive version

○ Weighted averages

- Partial least square regression

$\square$ Cross-validatory fit percentage $(>0)$

- Can be compared directly to that of predictive CCA (CCA-PLS)

- Randomization tests (MATLAB, R) 


\section{Additional methods}

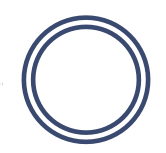

- A number of indirect approaches have been applied to evaluate crosstaxon congruence

- indirect gradient analysis and calculate pair-wise correlations of the resulting ordination axes (Hájek et al. 2002; ter Braak and Schaffers 2004)

- Bilton et al. (2006) used second stage NMDS (see Clarke et al. 2006) based on the Spearman rank correlation to calculate the similarity among different taxa in ponds

- Sætersdal et al. (2003):

o detrended correspondence analysis (DCA) on community data

- correlation of the results with a set of environmental variables using the Kendal rank correlation index 


\section{Biotic-abiotic relationships - 1}

- Species richness

○ Generalized linear models (GLM, Poisson, log-link)

○ Multiple regression model (FP; forward procedure described by Blanchet et al. 2008)

○ Distance-based linear models (DISTLM, Anderson 2006)

○ Multicollinearity:

* Pearson coefficient (0.60)

- Variance inflation factor (never $>10$ ) 


\section{Forward Procedure (FP)}

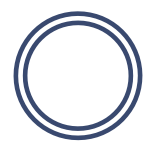

- This procedure is based on two stopping criteria:

$\circ$ 1) the significance level $\alpha$ (here set at 0.05)

○ 2) an adjusted coefficient of determination (maximum $R^{2}$ adj), calculated by constructing a full model inclusive of all explanatory variables

口 This forward procedure performs a forward selection by permutation of residuals under the reduced model

- Advantages:

$\circ$ 1) avoids the overestimation of the explained variance

○ 2) an inflated Type I Error 


\section{Biotic-abiotic relationships - 2}

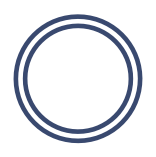

- Species composition

○ FP

○ Predictive CCA (CCA-PLS; ter Braak \& Schaffers 2004)

$\square$ Predictors:

$\square$ 1) plant species composition

$\square$ 2) environmental variables

○ Explanatory CCA

○ DISTLM (distance-based linear model)

o Procrustes analysis (using the results of non-multidimensional scaling for biotic communities, and principal components analysis for environmental data, NMDS-PCA) 


\section{Plant and beetle assemblages}

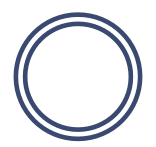

- Plant: 67 species

o Alpha diversity: 2-26

- Beetle: 76 species

○ Alpha diversity: 5-33 


\section{Cross-taxon congruence}

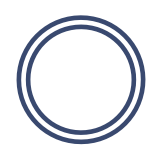

- Species richness: $\boldsymbol{r}^{2}=\mathbf{0 . 3 1 0 2}$

- Mantel test: $(\boldsymbol{r}=\mathbf{0 . 3 0 2}, P=0.001)$

- Permutational Mantel test (Spearman rank correlation rho $=\mathbf{0 . 3 3 7}$ )

- Procrustes analysis - PROTEST

○ Original data: correlation $(m): \mathbf{0 . 7 1 7}, P=0.001$

$\circ$ NMDS (3 axes): correlation $(m): \mathbf{0 . 5 6 8}, P=0.001$

$\circ$ Advantage: being based on the original matrices and not the distance matrix

o Higher power than Mantel tests 


\section{NMDS}

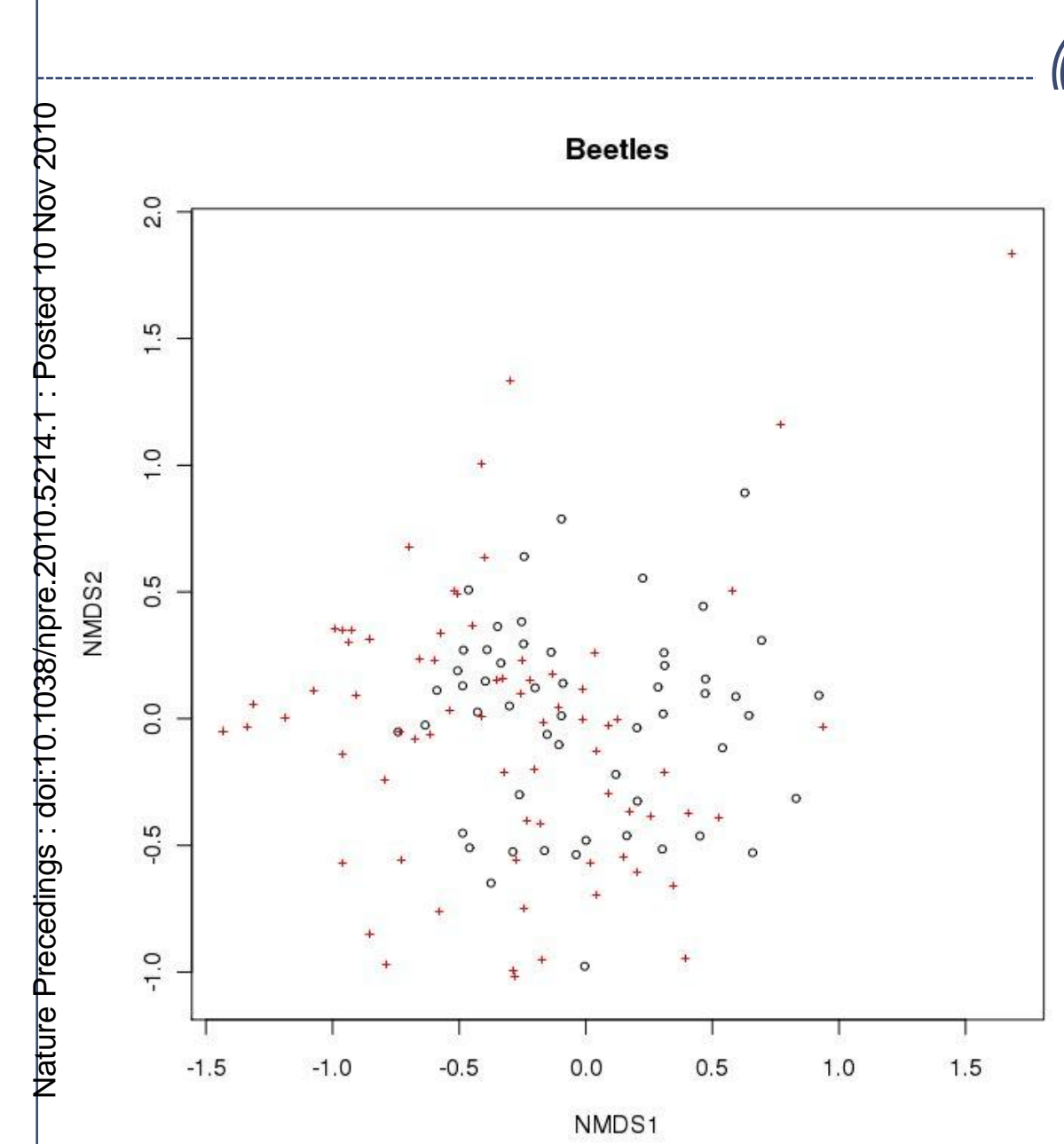

( )

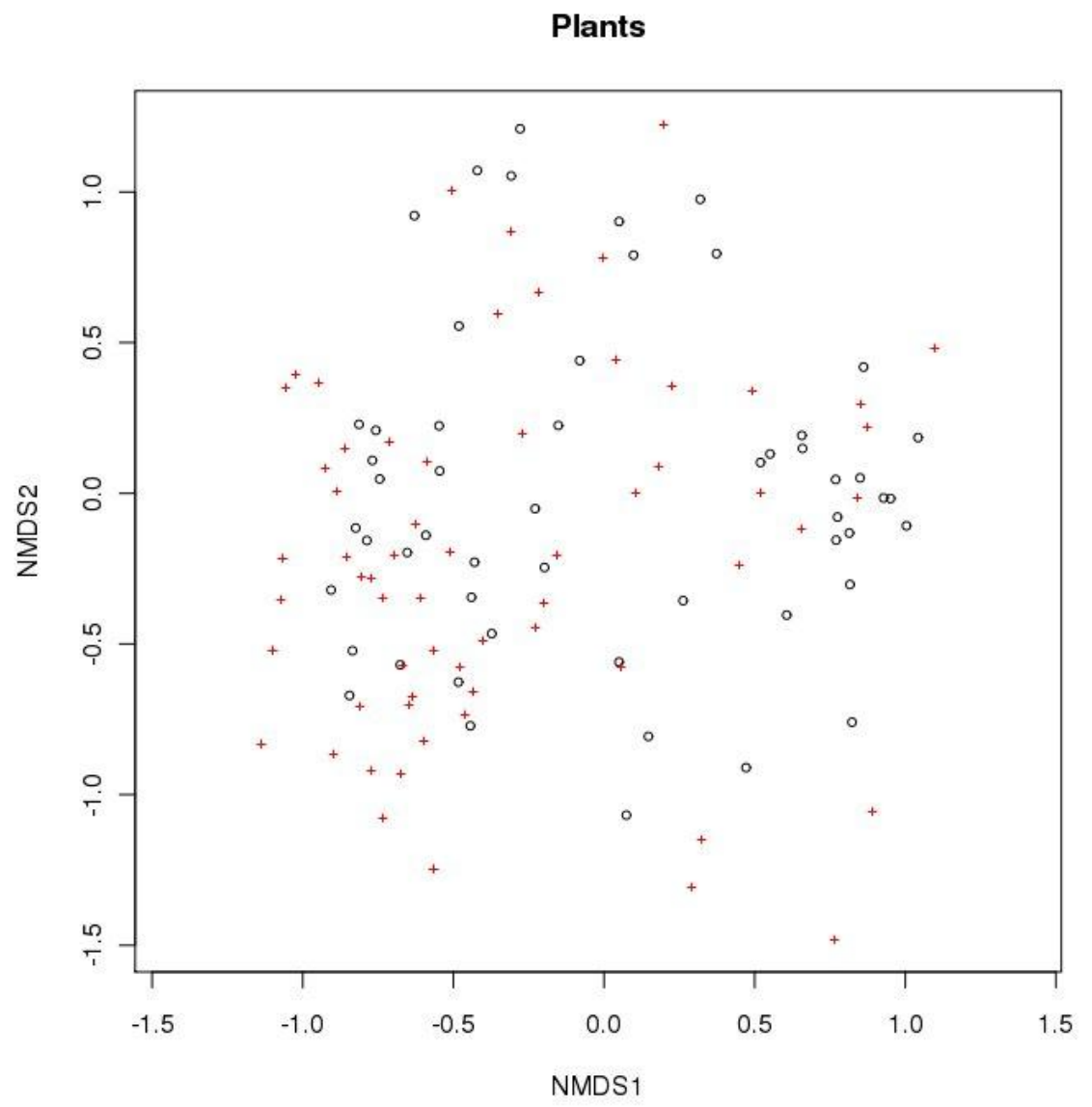

Fig. 1. Results of NMDS performed on plant and beetle community data collected from two regions from 54 farmland ponds 


\section{Procrustes analysis}

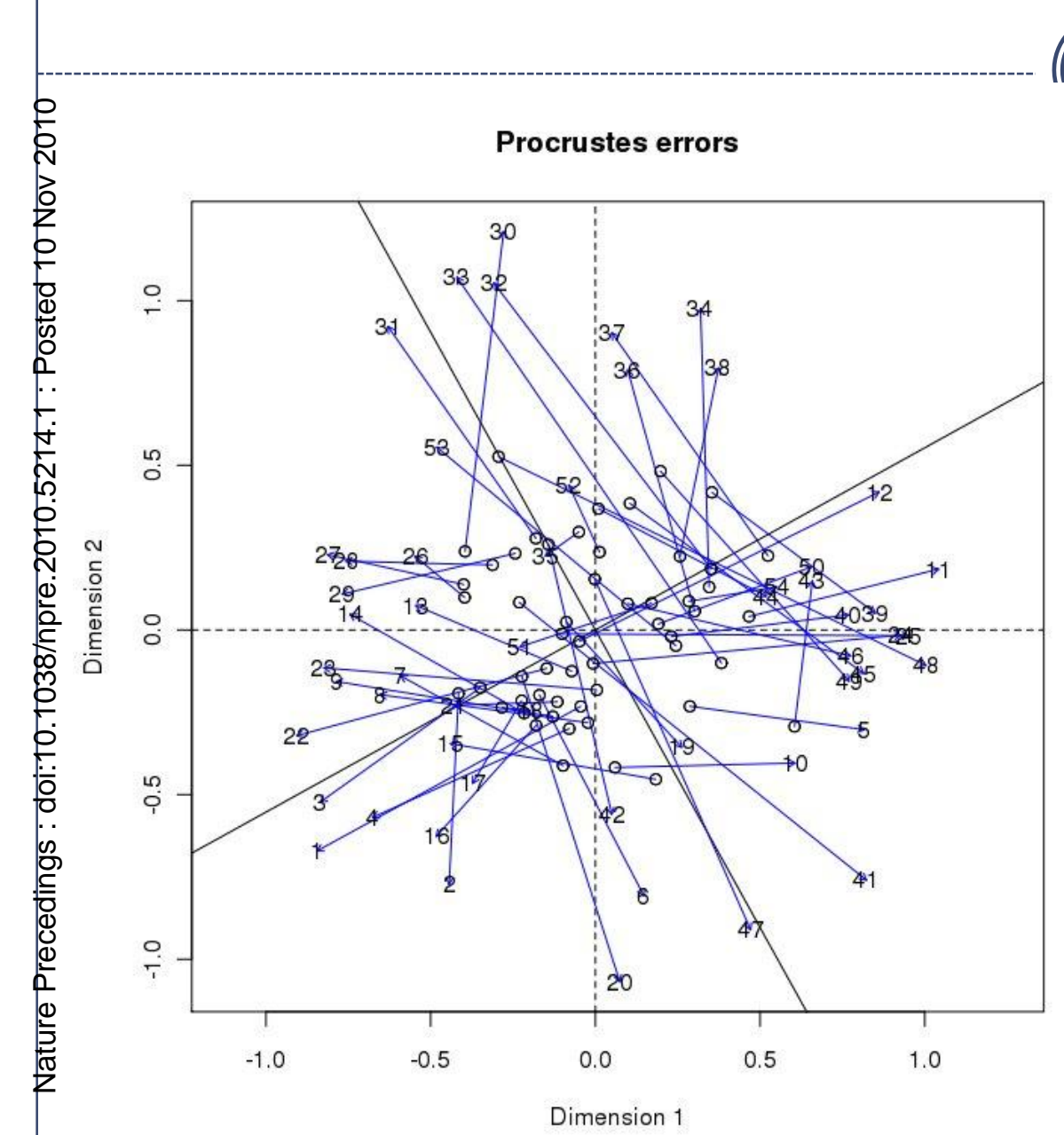

(1)

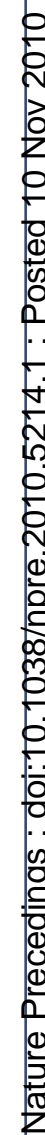

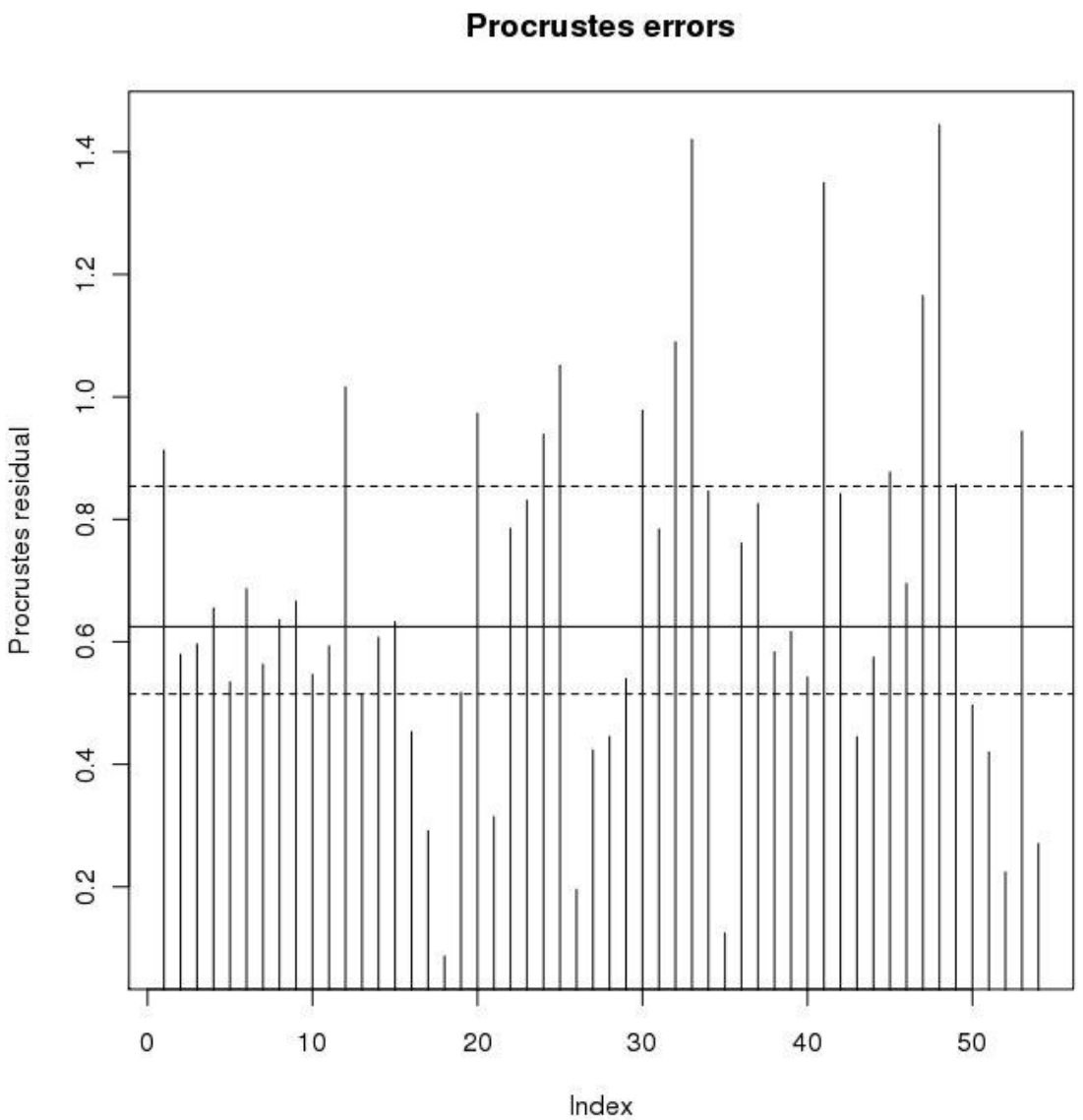

Fig. 2. Results of Procrustes analyses performed to evaluate the degree of correlation between plant (predictive) and beetle (response) assemblages collected from 54 farmland ponds 


\section{Predictive Co-CA and CCA-PLS}

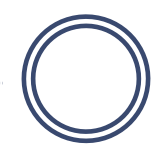

- Predictive Co-CA (Gioria et al. 2010, Biol Conserv.)

$\circ$ 1. Plant species composition (67): $\mathbf{5} \cdot \mathbf{3 4} \%$ variance $\left(2^{*}\right)$

$\circ$ 2. Environmental variables (12): $7.53 \%$ variance $\left(2^{*}\right)$

$\circ$ 3. Env. variables + Plant diversity $\left(J^{\prime}\right): 7.69 \%$ variance $\left(2^{*}\right)$

○ 4. Plant community type: $3.36 \%$ variance $\left(2^{*}\right)$

$\circ$ No statistical difference between plant species composition and environmental variables (Model 1 and 2)

- Symmetric Co-CA: 28.1\% explained variance 


\section{Predictive Co-CA}

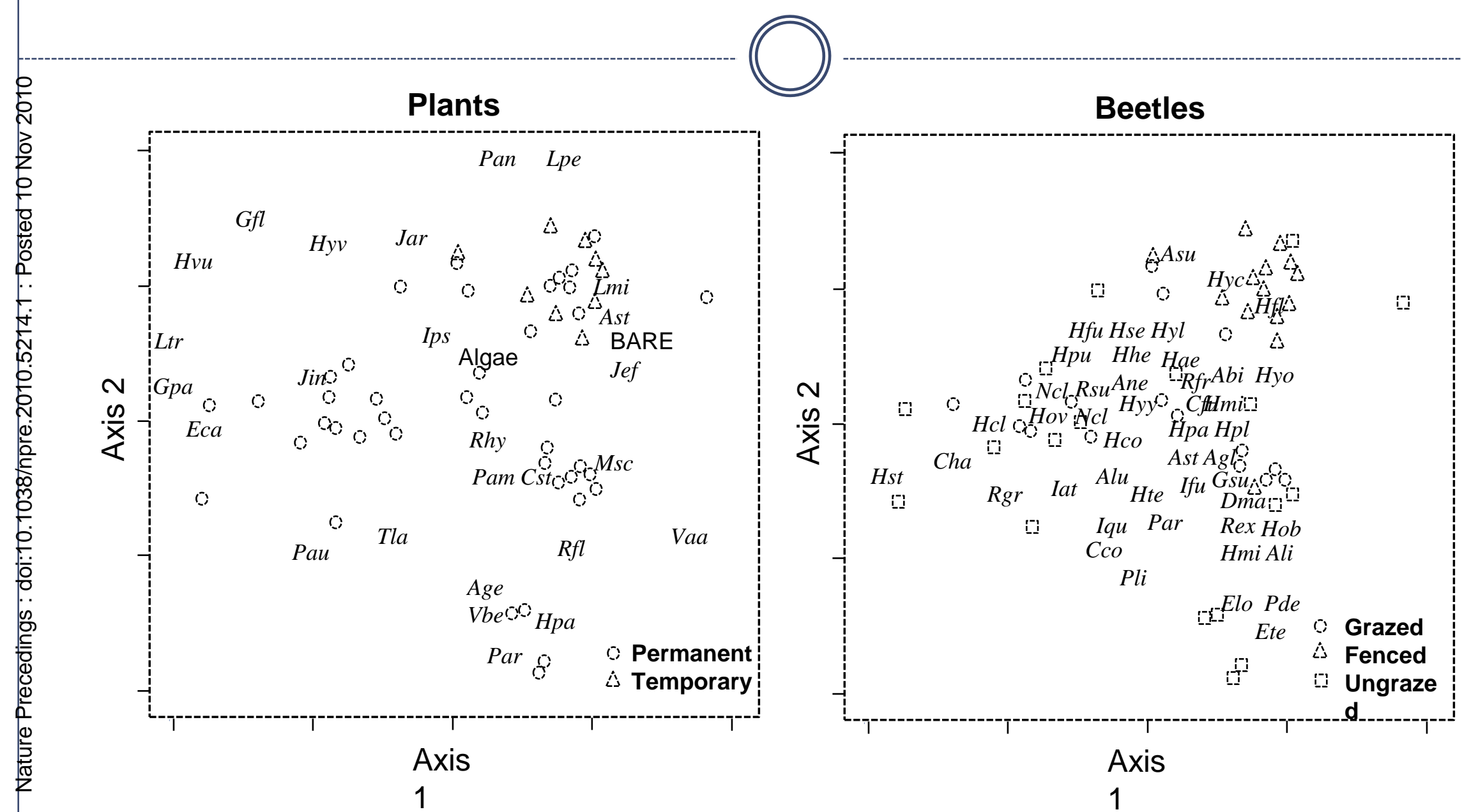

Fig. 3. Results of Co-correspondence analysis performed to evaluate the degree of correlation between plant (predictive) and beetle (response) assemblages collected from 54 farmland ponds 


\section{Biotic-abiotic relationships}

O

- Both plants and beetles responded in a similar way to the same set of environmental variables (depth, substratum)

- Identified a common set of environmental variables

- Selected a different number of variables (CCA)

o Different variables

o Contribution of each variable differed

○ Transformation

○ Measure of dissimilarity

○ Model selection criterion (AIC vs. BIC)

o Parametric vs. non-parametric

○ Species richness vs species composition (Gioria et al., Community Ecology) 


\section{Results biotic-abiotic relationships}

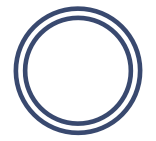

- GLM

- Beetle species richness

$$
\begin{array}{ll}
\mathrm{AIC}, D_{a d j}^{2}=0.727 ; & \mathrm{BIC}, D_{a d j}^{2}=0.697 \\
\mathrm{AIC}, D_{a d j}^{2}=0.565 ; & \mathrm{BIC}, D_{a d j}^{2}=0.538
\end{array}
$$

- DISTLM

- Beetle species richness: $R^{2}=0.730 ; \quad$ Beetle species composition: $R^{2}=0.408(0.305)$

- Plant species richness: $R^{2}=0.588 ; \quad$ Plant species composition: $R^{2}=0.305(0.215)$

- Forward Procedure

- Beetle species richness: $R^{2}=0.634 ; \quad$ Beetle species composition: $R^{2}=0.332$

- Plant species richness: $R^{2}=0.478 ; \quad$ Plant species composition: $R^{2}=0.184$

- CCA

- Beetle species composition: $\chi^{2}=0.469$

- Plant species composition: $\chi 2=0.193$ 


\section{Issues}

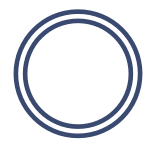

- Approach used substantially affect the results

- Species richness vs community composition (abundance)

- Parametric vs non-parametric

- Which method to measure cross-taxon congruence?

- Which method to model biotic-abiotic relationships?

- Use of multiple approaches

- Parametric vs non parametric

- Abundance data should be used 


\section{Recommendations}

- Experimental studies

- Balanced designs - use of categorical variables

- Protocol or analytical framework:

- $\rightarrow$ Meta-analysis studies 


\section{Biological conclusions}

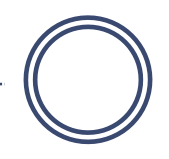

- Plant were a good surrogate group for water beetles in pond biodiversity assessments

- Plant species composition - good predictor of water beetle assemblages patterns

- Plants and water beetles respond in a similar way to the same environmental gradients (based on a range of ecological models) 


\section{Biological conclusions}

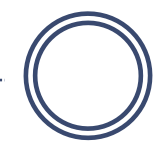

- Synthesize information on the biotic and abiotic conditions at a site

- Plant diversity - predictive value minimal - no suitable to predict community patterns

- Plant species composition - similar contribution than plant community type

- ? Redundant information - microhabitat provided, food, shelter, oviposition site

- Information at the species level only important for host-specific species or genera 


\title{
Acknowledgments
}

\author{
John Feehan (University College Dublin)
}

Burkart Dieterich (University College Dublin)

EPA Ireland - STRIVE Fellowship 